\title{
Application of BIM Technology in Construction of Visual Simulation
}

\author{
Binjie Qian", a, Liyuan Wang1, b, Xiaotong Cui1 ${ }^{1, c}$, Liu Chao*1,d \\ ${ }^{1}$ School of Environmental and Municipal Engineering, Qingdao University of Technology, \\ Qingdao, Shandong, 266033,China
}

a694617746@qq.com, b838979848@qq.com, c365319610qq.com, dliuchao.hy@163.com

Key words: Building Information Modeling, Construction Visualization, Simulate.

Abstract: With the building size, shape, function more and more complex, building systems have become more complex, often to a variety of complex piping systems are arranged in a limited space, and also to meet the appropriate space requirements. In order to achieve an intuitive understanding of the key parts of space conditions, and thus a reasonable layout of the respective tube, better use of the limited space, and effectively improve the design efficiency and quality, proposed construction BIM visualization techniques. In this paper, examples of a complex building construction for the study, the use of simulation software Revit building information model (building models and water drains Model) creation, combined Navisworks software for project construction visualization.

BIM technology can provide interacting and reaction visualizations between various construction components. The result of visualization can be used for architectural renderings exhibition and financial statements formation, moreover, all the communications, discussions, and decisions during project designing, construction, and operation are under an uniform visualized framework. As a result, architects can understand spatial arrangement in critical parts of project and take benefit of designing reasonable layout. Meanwhile, space utility rate and quality of project will also be improved by using BIM based technology.

This paper focus on a comprehensive building construction project which implemented based on visualized analysis model. The model is built by BIM technology-based Revit series software and visualized application analysis is under Autodesk Navisworks framework. These techniques can supervise actual project construction, furthermore, an example of BIM technology based project is provided to other enterprises.

\section{BIM technology analysis}

\section{Definition of BIM}

BIM is short for Building Information Model, which integrates relevant information in construction project together. In order to present details of project, 3D visualization and digitization of information technologies are employed [1]. Information representation problem is a critical issue in construction project while the implementation of BIM technology can help architects and engineers understanding relevant information. Moreover, with the assist of Building Information Model, they would come up with appropriate approaches and solutions. This will significantly enhance the teamwork during the construction project.

Implementing BIM technology can achieve better construction quality, project efficiency, and cost reduction, which means more profits. This will lead construction information technology to a new era. 


\section{Features of BIM}

BIM based collaborative working environment is sustainable and transparent. In a such framework, it is possible and convenient to copy and inspect information of construction components. Therefore, the communications between project participants and information sharing are agile and convenience. Various participants could work together according to comprehensive information provided by BIM technology and efficiently manage projects. There are some unique features compared with traditional methodology: 1 . design visualization, architects can completely understand space clearance in critical parts and design reasonable layouts which increases utility rate and quality of the design; 2. collision detection, BIM technology provides an inspection for space clearance to detect if building structure, pipeline, and other components are conflicting. Meanwhile, timely and appropriate adjustments would be performed when collision happens; 3. uniform model, comprehensive construction information converges to a uniform model, that means when design changing happens, only modification operations on the model is required; 4. information sharing, BIM technology collects project statements in multiple subjects and sharing them by using linking model method[2].

\section{Comparison between BIM and traditional CAD}

In traditional CAD technology based project, 2D architectural and other drawings are the most critical foundations. However, there are very weak correlations between various types of drawings, which always causes conflicts in the design process. Moreover, when a conflict happens, corrections and modifications on different subject drawings are extreme challenging.

Construction progress and schedule in design process are two critical points to differ BIM technology from traditional AutoCAD design. BIM technology is based on 3D models and operates project designing, construction drawing designing, design visualization, and even environmental simulation in an unified framework. Three major comparisons are described as follow [3]:

(1) 3D/4D (3D plus time dimension) parametric design

As mentioned before, traditional AutoCAD and other software are based on 2D design, even they enable 3D modeling functions while BIM can support professional 3D design tools, real-time $3 \mathrm{D}$ visualization, and advanced collaborative designing. Moreover, detailed construction drawings and specifications can be generated from model automatically. Representation of complicated models is a challenging issue in traditional CAD design, while BIM takes advantage of 3D parametric design. Furthermore, efficient solving complicated pipes arranging problems is much more easy in BIM based framework.

(2) Collaborative design and construction

Compared with CAD technology, BIM system guarantees entire operations of each stage and process during the life circle. It considers the entire process including planning, designing, construction, and operation. By implementing BIM based framework, project can avoid misunderstanding and lacking of communication in the designing process. Meanwhile, increasing the efficiency of designing and management, reducing cost, and achieving more profit are other advantages of BIM technology.

(3) Simulation, Optimization, and Output

In traditional AutoCAD framework, simulation and optimization processes only happen when proprietors require green building. Even though, the accurateness is not satisfactory in architecture and energy consumption aspects.

Nevertheless, BIM technology can highly simulate real world conditions, as a result, analysis of performance, energy consumption, daylighting, evacuating, and flow field can be operated. These 
data are extreme accurate and practical and the output based on 3D model outperforms CAD technology.

\section{Background and related work}

Nowadays, construction visualization is still developing and some enterprises have implemented BIM technology in project. For example, IES has developed a visual environment system for construction designing; Shimizu corporation generates construction information by using 3D CAD technology. Moreover, shanghai zhengda plaza project has implemented BIM technology in the steel structure installation. The successful implementation during the project provided high quality, safety, and perfect efficiency.

Current BIM technology has three features: first, the research on construction theory is basement of construction visualization; second, visualized simulation, lean construction, and agile construction can support visual construction; third, software and system development enhances BIM technology and help popularization [4].

The implementation of construction visualization is a highly strong requirement in future building industry and causes revolution of construction mode. However, there is still long way to get to future digital construction and this paper will explore and research BIM based modeling in construction project $[5,6]$.

\section{BIM modeling}

\section{Software and hardware environment}

The machine used for modeling is based on Intel Core i7-4820K CPU with 32GB RAM and running Microsoft Windows 7 Ultimate 64 bit. Autodesk Revit 2013 series including Revit Architecture and Revit MEP are employed for 3D construction modeling.

Information modeling is the startup process because BIM model is a central database for data communication and sharing. 3D design software works with simulation software and provide both comprehensive and accurate system.

This paper focus on an example of comprehensive building construction project. Especially, ratio of reinforcement and loading analysis are out of boundary of this paper. By using Revit Architecture, major structure of the building is designed and generates BIM model. Moreover, water supply and drainage subject information is handled by Revit MEP software.

\section{BIM based Modeling}

(1) Structure modeling

From whole to parts is the principle to building models in Revit Architecture. After choosing project template, elevation and axis nets should be defined. The next step is using software to create building walls, doors, windows, floors, and rooftop. Moreover, the software supports ground and terrace setup and other components. Based on the model, other details like dimension markings and comments should be defined. After view adjustment, drawing printing, and model rendering, other analysis and design software will join the construction for interacting.

When create project in Revit Architecture, every project is based on template. Various components and parameters are added to the template. As is shown in Figure 1 and Table 1. 


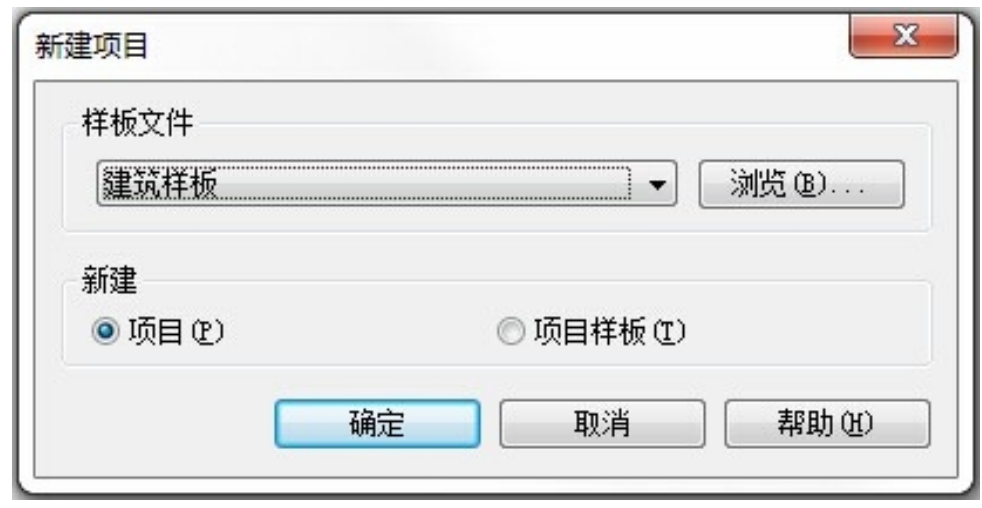

Figure 1 Construction model of the project

Table 1 Construction model of the project

\begin{tabular}{|c|c|c|}
\hline \multicolumn{3}{|l|}{ New project } \\
\hline \multicolumn{3}{|l|}{ Boilerplate } \\
\hline Building model & & \\
\hline \multicolumn{3}{|l|}{ Create } \\
\hline Project & \multicolumn{2}{|c|}{ Project template } \\
\hline $\mathrm{OK}$ & Cancel & Help \\
\hline
\end{tabular}

The first step of modeling in Revit Architecture is to define height, direction, and spatial position, which are elevation and axis net. Figure 2 and figure 3 shows how to setup them, in addition, they are precondition and basement of collaborative design in Revit framework.

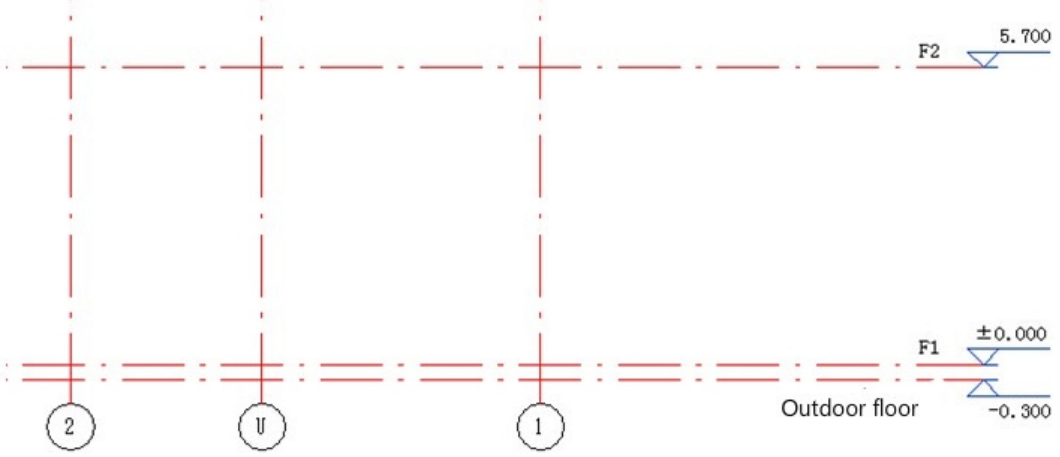

Figure 2 Elevation drawing and number

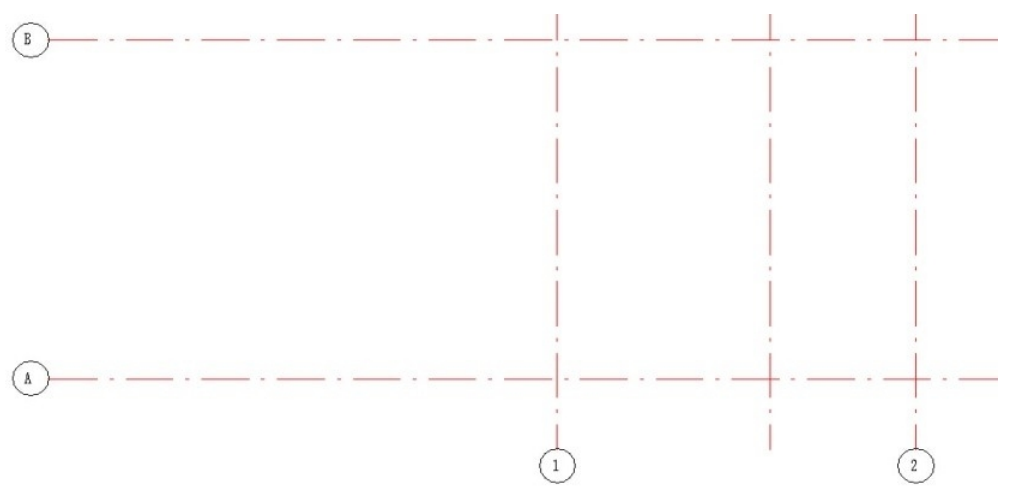

Figure 3 Axis net drawing and number 
Then, the second step is creating walls, doors, windows, floor, and other components of the building. At last, rendering function could actualize the model and generate BIM model shown as figure 4.

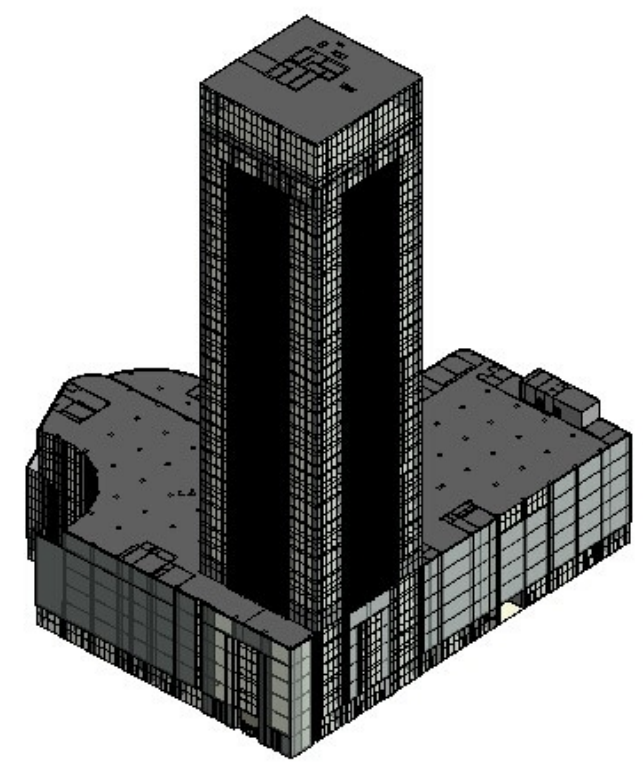

Figure 4 Main model of BIM construction

(2) MEP modeling

As well as creating building template, project in Revit MEP is also based on template. In this section, mechanical template is chosen. After setup project information, structure model will be linked to the project file. There are two kinds of elevation which are linked structure elevation and template elevation. It is obvious that linked structure elevation should be used and prepared before project designing. After preparation, water supply and drainage designing could be implemented in the BIM framework, which are fire hydrant system and automatically sprinkling system.

Before designing pipeline of fire hydrant, types and attributes of pipeline should be defined. Then fire hydrant box class is loaded to the project and arranged to the appropriate positions. According to the positions, fire control stand pipes are setup and connect to fire hydrant boxes. After connect horizontal pipes, water supply system designing is finished. Figure 5 shows fire hydrant system.

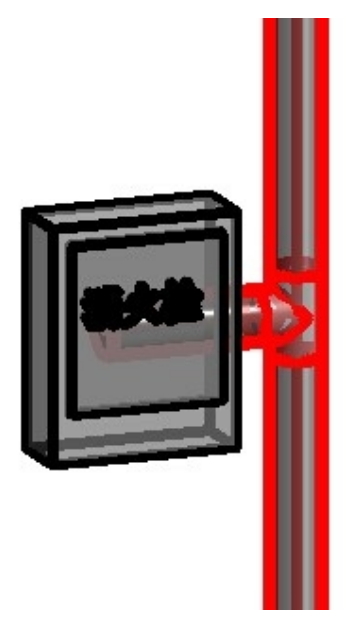

Figure 5 Fire hydrant system

It is extreme similar with hydrant system, automatically sprinkling system also requires setup types and attributes of pipeline. Based on separation distance requirements, sprinkling nozzles are 
located and connected. Figure 6 shows final 3D model in BIM framework.

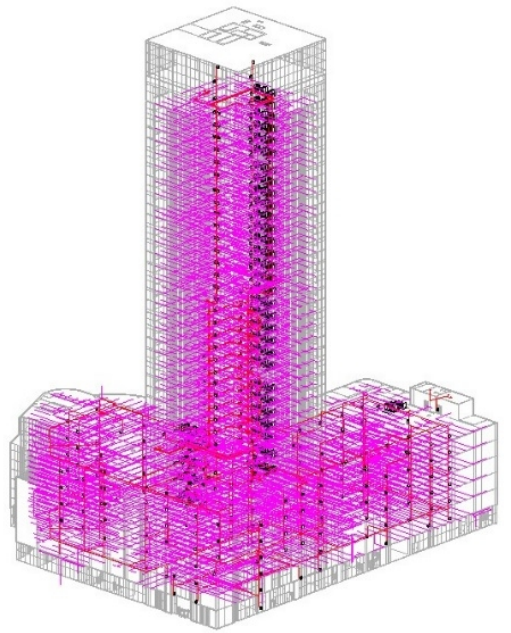

Figure 6 The final 3D model of the project

\section{Operation of BIM construction visualization}

\section{Software platform of visualized simulation}

When the BIM model of general building is finished, it could be loaded in the Autodesk Navisworks. This software enables 3D simulation during construction process based on BIM model, which can support subsequent construction for better management. The model integration function in Navisworks allows users choosing appropriate software to build up 3D model. Moreover, it is real time visualization and enable exploring in complicated 3D model [7].

\section{Construction visualization process}

After creating task in BIM model, Timeliner funtion in Navisworks Manage software is employeed for connecting construction progress. The implementation of construction visualization is described as follow:

The first step is to use Timeliner command creating task, then properties of the task and Gantt chart should be adjusted. After setup is finished, construction simulation model is built.

Furthermore, it is important and necessary to confirm related parameters based on construction missions. The result will output for future works when simulation is finished.

\section{Result}

The result of construction simulation implementation is based on professional modeling tools and shown in figure 7. 


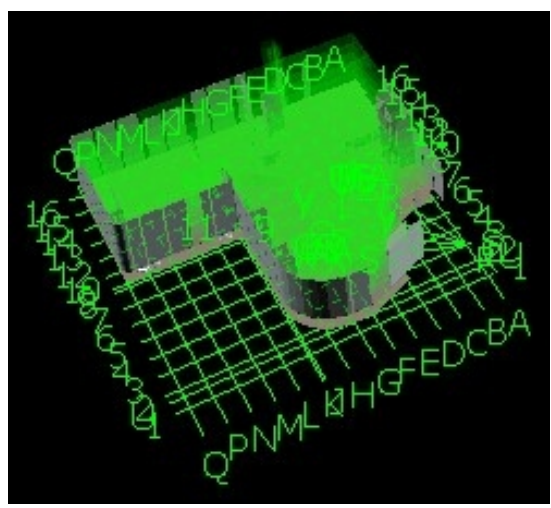

(1)

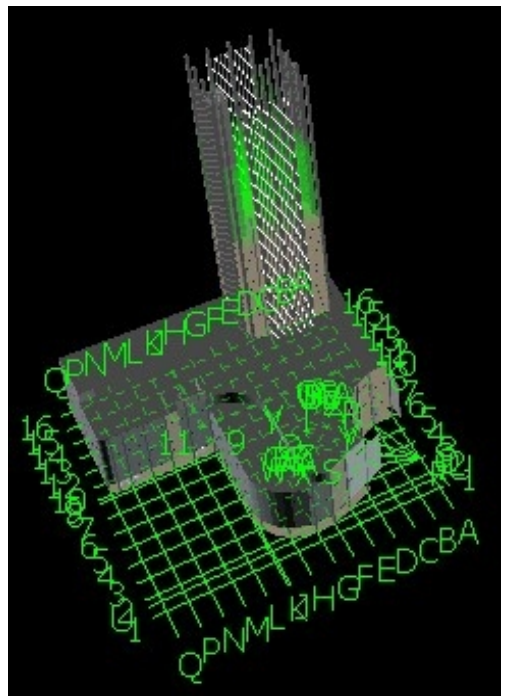

(3)

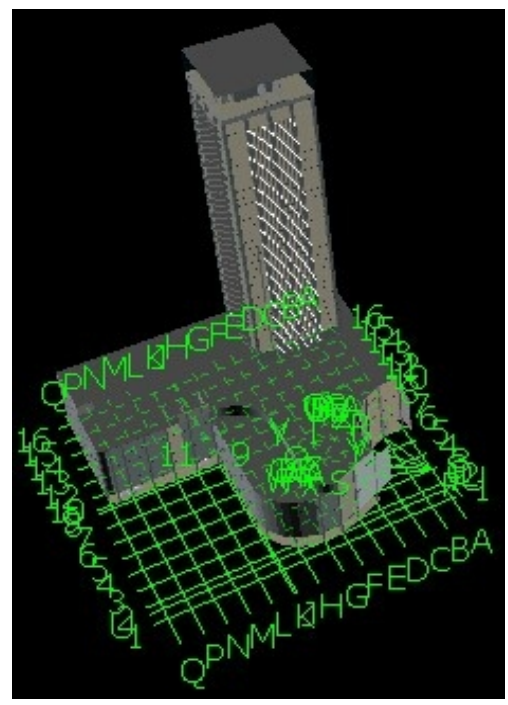

(4)

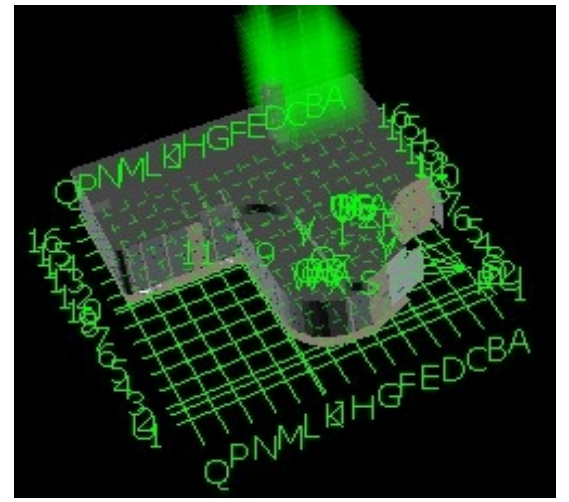

(2)

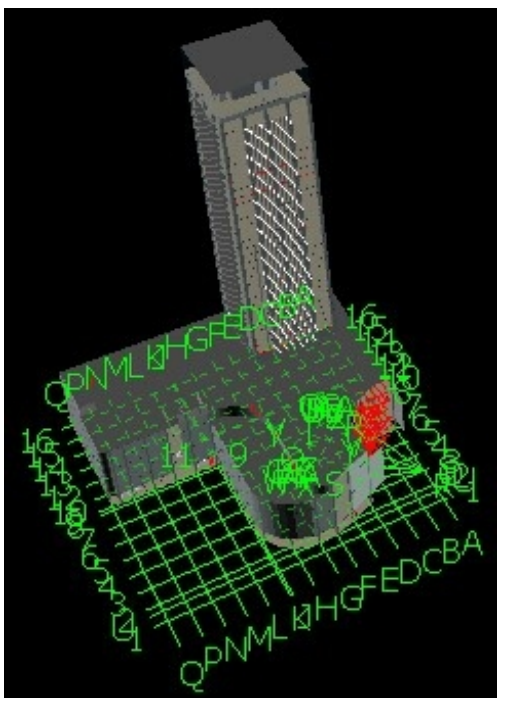

(5)

Figure 7 Result of construction simulation

\section{Implementation of BIM construction visualization}

\section{BIM model based 3D drawings review}

In traditional 2D review process of the construction drawings, it is necessary to review the plan, section and elevation drawings of each profession of building, structure, HVAC, water supply and drainage, electrical, fire individually. The workload is really heavy. In addition, due to the difficulties of communication between various professions, the review is not comprehensive and accurate enough, which causes many problems in the construction phase of the project, leads to reworks, and severely reduces the efficiency of the construction.

Using visualization platform of BIM to make the artifacts in 3D model to take the place of lines, marks and text notes in the 2D construction drawings. In BIM models, it has a clear view to help finding problems. It doesn't need to review other 2D drawings, which reduces the workload and makes it easy to find the problems existing in the design, reduced the difficulties of drawings review. In addition, the use of BIM visualization technology after finding the problems, makes it easier for various professions to make modifications reciprocally, and effectively avoids the imperfection of the modifications caused by the communication difficulties in the traditional $2 \mathrm{D}$ construction drawings reviewing, improved the quality and efficiency of drawings review.

\section{Analysis of construction progress}

Crossing figure technology is a common method in traditional projects of construction progress. 
This method uses the form of tables and drawings to make expression, which is simple, clear, easy to learn. Moreover, it is easy to check and calculate the resource requirements of the project. However, it still exists some limitations which mainly shows in the following aspects:

(1) Using tables and drawings to express each stage of construction progress plan in the project, can not accurately reflect the logical relationship between each work. That leads to irrationality before and after the construction plan.

(2)It can't reflect the critical factor in construction plan, which makes the contractors in construction plan have no emphasis.

(3)When the project is changed or something unexpected happens in the process of the construction, the construction progress which specified by traditional crossing figure technology can't change with it, it only can be modified, and even needs to remake the whole construction progress plan.

(4)The traditional construction progress plan cannot use the modern computer technology to optimize, which increases workload when choosing the optimum construction scheme.

Compared with the traditional crossing figure technology, the use of construction visualization technology based on BIM to conduct construction simulation can not only find the problem before the construction of the project, but also can help each project participants analyze and solve problems. Using 3D building information model to link with construction progress plan to conduct construction simulation can make analysis and comparison for different construction plans, thus, to choose the optimal solution. Meanwhile, it is much more convenient when analyze the root causes of some construction problems, such as quality deviation in construction plan and schedule delays, so as to take corresponding solutions in time.

In addition, in the process of construction of the project, the owners or design companies often change the project because of all sorts of problems. According to the owners or design companies' modifications, using the construction visualization technology can predict their will, which makes the changed plan's influence on the subsequent construction more intuitive and clear, so as to provide support and basis for the owners' decisions.

\section{Optimization of Integrated pipeline arrangement}

In the traditional design process, the design between the various professions is separated from each other, and most of the design is two-dimensional design. Due to lack of logical relationship between each work, it is need to integrate all the professional engineers together and imagine its threedimensional shape when the project is checked. The check is difficult and the result is not comprehensive and accurate. During the construction process, it often occur collision between the building structure, equipment pipelines collision between the professional and the collision between the building structure and pipelines.

BIM technology will use the building, structure, water supply and drainage, HVAC, electric, fire and other integration among different profession models to optimize integrated pipelines arrangement, all participants can found the unreasonable of design in advance and timely resolve to provide the most optimized model for the construction project. Mainly in the following areas:

(1) Using BIM technology to integrate the professional 3D model, and then uploaded to the Navisworks software platform for its collision check, you can automatically find out the collision point of the model, so that the collision is visual and through the "Image + Text "type description output paper reports.

(2) To upload the 3D building information model to BIM system, so as to accurately observe the project specific configuration of complex pipeline node generates a detailed two-dimensional to guide 
the subsequent construction.

(3) To achieve internal roaming by BIM model before the construction of the project, and to observe the internal arrangement of pipelines directly, to optimize the arrangement of pipelines, so as to avoid the problems encountered in the construction process, reduce rework [8].

\section{Conclusion}

BIM technology with time dimension can implement construction disclosure, visualization, and finding difficult building components. With respect to construction standard, BIM model helps scheduling progress and modeling project. As a result, the ultimate goal is to increase quality and manage cost.

\section{References}

[1] Liu Zhaoqiu, Li Yungui. The development of building information model and its application in design [J]. Building Science, 2009 (1): 96-99.

[2] He Guanpei, Li gang. What changes will BIM application also bring to buildings [J]. Information Technology, 2010 (01): 10 to 17.

[3] Ren Bo. From 2 D to 3 D computer aided architectural design [D]. Chongqing University, 2010.

[4] Liu Juanhua, Li Yanni. Virtual construction technology based on BIM application.Computer Knowledge and Technology, 2011, 7 (29) : 7266-7268.

[5] YueJie. BIM and its application in architectural design [J]. Journal of Building Materials in Sichuan, 2011, 5 (37): 270-271.

[6] Hou Xiaoting, Li Changhua, Lai Bingheng. Key techniques of virtual construction [J]. Mechanical Science and Technology, 2011, 30 (7): 1196-1201.

[7] Zhang Li, Shi Yi. Application practice,research and development prospects of virtual construction technology [J]. Industrial Construction, 2003 (11): 49-51.

[8] Liu Yongkui, Zhou Xiaomin. Virtual reality technology and scientific computing visualization, Chinese Journal of Image and Graphics, 2000, 5 (9): 794-798. 